\title{
Optimal and Adaptive Battery Discharge Strategies for Cyber-Physical Systems
}

\author{
Fumin Zhang and Zhenwu Shi
}

\begin{abstract}
We introduce a dynamic battery model that describes the variations of the capacity of a battery under time varying discharge current. This model is input-output equivalent to the Rakhmatov-Vrudhula-Wallach (RVW) model that has been verified by experimental data. Our model allows battery capacity prediction for feedback control laws and online scheduling algorithms that are building blocks for CyberPhysical Systems (CPS) theory. We design optimal and adaptive discharge profile for a square wave impulsive current to achieve maximum battery life. Simulation results are provided to compare battery life for different discharge profiles.
\end{abstract}

\section{INTRODUCTION}

Technology advancement in battery design has played an important role in the economic development during the last two decades. Consumer electronics, mobile computing devices, and personal communication devices have become smaller but with longer operation time. Battery innovation also finds its way into the research community in areas such as robotics, mobile sensor networks, and embedded computers where most hardware platforms are powered by batteries. Until now, the majority of batteries used are electro-chemical batteries. Such batteries are complex chemical/physical systems by themselves and possess interesting "nonlinear" discharge behaviors [1]-[3].

A recent trend in cyber-physical systems research is the co-design of different controlling mechanisms to balance performance in both physical systems and computing systems [4]-[8]. The results of such co-design are often hybrid feedback control laws and scheduling algorithms that draw impulsive current from the batteries. Approaches for power management have been proposed, with the method of dynamic voltage scaling (DVS) among the most important ones. Reference [9] proposes one of the first DVS-aware scheduling algorithms, [10] analyzes DVS scheduling with discrete voltages, [11] develops heuristic schedulers that take advantage of program profile information, [12] introduces procrastination scheduling, and [13] manage power for sensor networks. In these works the behaviors of batteries are simplified as ideal voltage sources whose life only depends on the average discharge current.

According to battery and VLSI design literature e.g. [1], [2], the battery discharge current is supported by the change of concentration of electrolytes near the anode or the cathode of a battery. The chemical processes of oxidation

The authors are with School of Electrical and Computer Engineering, Georgia Inst. of Technology, Savannah, Georgia 31407. Email: \{fumin, zwshi\}@gatech.edu. We would like to thank Dr. Wayne Wolf and Dr. Xue Liu for discussions. The research is supported by ONR grant N00014-081-1007 and NSF grant 0841195. and reduction reduce the concentration of electrolytes near the electrodes and create a spatial gradient of concentration within the battery. Driven by the concentration gradient, electrolytes diffuse to the electrodes from other areas of the battery. When the concentration at the electrodes drops below a certain threshold, a battery fails to provide discharge current, causing failure to devices it supports. At this moment, the discharge cycle has to stop and the battery needs to be recharged or replaced. Note that there may be a significant amount of active electrolytes left in the battery when a discharge cycle ends. Therefore, the actual capacity of a battery is significantly lower than the theoretical capacity that is the total amount of electrolytes contained in a battery. When the battery is discharged under a pulsed discharge current, during the idle time when current is interrupted, the diffusion process increases electrolyte concentration at the electrodes. This produces the recovery effect that makes the battery appears to have regained portions of its capacity. The amount of capacity recovered depends on the amount of active electrolyte available.

Battery modeling aims to simulate these behaviors by computational models [2]. To support theoretical cyberphysical systems design, a class of analytical models are desired. This is because comparing to physical [14], empirical [15], and circuit based [16] models, analytical models are theoretically tractable while providing sufficient accuracy. Authors of [1] model battery behaviors as a stochastic system based on random walks on a finite state machine. Authors of [17] model the nonlinear relationship between battery capacity and averaged discharge current. The RakhmatovVrudhula-Wallach (RVW) model proposed in [3] is an analytical model that is derived from solving the diffusion equations governing the electrolytes motion within a battery. The model captures the recovery effect effectively. It has been shown to agree with experimental results and has demonstrated high accuracy in battery capacity prediction and battery life estimation. However, the application of the RVW model requires that the discharge current, as a function of time, is known. This is because the RVW model is typically used at the circuit design stage to evaluate design options on battery discharge.

A dynamic model needs to be established that is able to predict battery capacity based on the discharge current determined by a feedback control law or feedback scheduling algorithm. The magnitude and pulse width of such discharge current can not be determined beforehand. In this paper, we establish a battery model that captures the recovery effect and supports the co-design of battery management with con- 
troller and scheduling algorithms. Our model is input-output equivalent to the RVW model. Based on this model, optimal and adaptive discharge profiles for square wave discharge current is determined. We show that the problem is not more challenging than studying linear systems under impulsive or switching control. Although such systems are mostly wellunderstood theoretically in the control community [18]-[20], our effort connects these known results on systems behavior with battery behaviors, which is a novel contribution.

The paper is organized as follows. We review the electrochemical battery discharge mechanisms and the RVW model in section II. In section III, we derive the dynamic battery model. The model behavior under a square wave impulsive discharge current is discussed in section IV. The optimal impulsive and adaptive discharge profile is computed in section VI. In section VII, we compare battery life for different battery discharge profiles via simulation. Conclusions are provided in section VII.

\section{BAtTeRy Mechanism AND THE RVW MODEL}

This section reviews the relevant properties of electrochemical battery cells and the RVW model. We summarizes the discussions in [1]-[3] and the references therein.

\section{A. The Electrochemical Process}

An electrochemical battery has a cathode and an anode that are connected by electrolyte inside the battery shell. During discharge, the gain of electrons at the cathode (oxidation) is coupled with the loss of electrons at the anode (reduction). The electrochemical reactions, involving electrons $v^{-1}$,oxidized species $O$, and reduced species $R$, can be described as follows:

$$
\begin{gathered}
\text { cathode } O+v^{-} \rightarrow R \\
\text { anode } R-v^{-} \rightarrow O .
\end{gathered}
$$

To simplify the discussion, let us suppose that a battery is symmetric and the two electrodes behave in similar ways. This allows us to consider only species $O$ and the cathode.

Initially, the concentration of species $O$ is uniformly distributed inside a battery. During the discharge period, the species $O$ at the cathode accepts electrons from external circuit and forms species $R$. This causes reduction of $O$ near the cathode, which generates a concentration gradient of $O$ across the battery. Due to this gradient, the species $O$ that is further away from the cathode diffuses to the cathode. The diffusion tends to increase the concentration of $O$ at the cathode. Under constant discharge current, eventually, the diffusion and the consumption reach a balance at the cathode, and the overall concentration of $O$ at the cathode keeps dropping. Once the concentration falls below a certain level, the battery fails to support the outside circuit.

If load is switched off before the battery fails, the cathode fails to draw electrons from the external circuit and thus the transformation at the cathode (from species $O$ to species $R$ ) stops. However, the concentration gradient inside the battery still exists and the diffusion will continue until the concentration gradient disappears. Now species $O$ will become uniformly distributed at a level lower than the initial concentration. Hence, given the same averaged current, the amount of charge delivered under the impulsive current is larger than the amount of charge delivered under the constant current.

\section{B. The RVW Model}

Rakhmatov, Vrudhula, and Wallach studied a onedimensional diffusion equation that describes the concentration of electrolytes inside a battery:

$$
\begin{aligned}
J(x, t) & =-D \frac{\partial C(x, t)}{\partial x} \\
\frac{\partial C(x, t)}{\partial t} & =D \frac{\partial^{2} C(x, t)}{\partial x^{2}}
\end{aligned}
$$

where $C(x, t)$ stands for the concentration of electrolyte at time $t$ at the distance $x$ from the electrode, $J(x, t)$ denotes the flux of electrolyte and $D$ is the constant diffusion coefficient. Suppose the length of the battery is $2 w$. Then the boundary condition for (1) can be derived as,

1) $x=0$ : according to Faraday's law, the flux of electrolyte $J(0, t)$ at the electrode is proportional to the current $i(t)$ supplied by the battery.

$$
-J(0, t) \nu A F=i(t)
$$

where $F$ stands for the Faraday Constant, $A$ stands for the cross section of the electrode, and $\nu$ is a scaling factor. One can derive the first boundary condition as

$$
\left.D \frac{\partial C(x, t)}{\partial x}\right|_{x=0}=\frac{i(t)}{\nu F A} .
$$

2) $x=w$ : the concentration gradient at the center of the battery is zero. Thus, one can derive the second boundary condition as

$$
\left.D \frac{\partial C(x, t)}{\partial x}\right|_{x=w}=0 .
$$

To solve (1), the Laplace transform method is applied. Since the electrolyte is uniformly distributed when discharge started, $C(x, 0)$ is a constant and can be denoted by $C^{\star}$. The solution for the concentration of electrolyte at the electrode is

$$
C(0, t)=C^{\star}-\frac{i(t)}{\nu w F A} *\left(1+2 \sum_{m=1}^{\infty} e^{-\frac{\pi^{2} m^{2}}{w^{2}} D t}\right)
$$

where $*$ stands for the convolution. Define $\rho(t)$ as

$$
\begin{aligned}
\rho(t) & =1-\frac{C(0, t)}{C^{\star}} \\
& =\frac{i(t)}{C^{\star} \nu w F A} *\left(1+2 \sum_{m=1}^{\infty} e^{-\frac{\pi^{2} m^{2}}{w^{2}} D t}\right)
\end{aligned}
$$

At the starting time $t=0, C(0,0)=C^{\star}$ and $\rho(0)=0$. As the discharge continues, $C(0, t)$ gradually decreases and $\rho(t)$ increases. Finally at the dead time $t=L, C(0, L)$ drops to the cutoff level $C_{\text {cutoff }}$ while $\rho(t)$ reaches the corresponding threshold $\rho_{\text {cutoff }}=\left(1-\frac{C_{\text {cutoff }}}{C^{\star}}\right)$. Note that both $\rho_{\text {cutoff }}$ and $C_{\text {cutoff }}$ are constants determined by the physical property of a given battery. 


\section{A Dynamic Discharge Model}

The RVW model relates the concentration at the electrode with the discharge current $i(t)$. But the relationship is static in the sense that the current $i(t)$ must be known for the entire discharge cycle. We present a state space dynamic model that produces the same input-output relationship as the RVW model.

Let $\lambda_{m}=\frac{\pi^{2} D}{w^{2}} m^{2}$ and $\alpha=C^{\star} \nu w F A \rho_{\text {cutoff. Then (5) }}$ becomes

$$
\rho(t)=\frac{i(t) \rho_{\text {cutoff }}}{\alpha} *\left(1+2 \sum_{m=1}^{\infty} e^{-\lambda_{m} t}\right)
$$

To normalize $\rho(t)$, we define $y(t)$ as

$$
y(t)=\frac{\rho(t)}{\rho_{\text {cutoff }}}=\frac{i(t)}{\alpha} *\left(1+2 \sum_{m=1}^{\infty} e^{-\lambda_{m} t}\right)
$$

which implies that $y(0)=0$ at the initial time $t=0$ and $y(L)=1$ at the failure time $t=L$.

To derive the state space model, we first replace $m=\infty$ with $m=M$ in (6) and obtain

$$
\begin{aligned}
y(t) & =\frac{i(t)}{\alpha} * 1+\frac{i(t)}{\alpha} * 2 \sum_{m=1}^{M} e^{-\lambda_{m} t} \\
& =\left[\begin{array}{llll}
1 & 1 & \cdots & 1
\end{array}\right]\left[\begin{array}{c}
\frac{i(t)}{\alpha} * 1 \\
\frac{2 i(t)}{\alpha} * e^{-\lambda_{1} t} \\
\cdots \\
\frac{2 i(t)}{\alpha} * e^{-\lambda_{M} t}
\end{array}\right]
\end{aligned}
$$

Next, we define the state variables to be $x(t)=$ $\left[x_{0}(t), \ldots, x_{M}(t)\right]^{T}$. The elements of $x$ satisfy,

$$
\begin{cases}\dot{x}_{0}(t)=\frac{1}{\alpha} i(t) & \\ \dot{x}_{m}(t)=-\lambda_{m} x_{m}(t)+\frac{2}{\alpha} i(t) & m \in\{1,2, \cdots, M\} \\ x_{m}(0)=0 & m \in\{0,1, \cdots, M\}\end{cases}
$$

The solutions of the above equations are,

$$
\left\{\begin{aligned}
x_{0}(t) & =\int_{0}^{t} \frac{1}{\alpha} i(\tau) d \tau=\frac{i(t)}{\alpha} * 1 \\
x_{m}(t) & =\int_{0}^{t} e^{-\lambda_{m}(t-\tau)} \frac{2}{\alpha} i(\tau) d \tau \\
& =\frac{2 i(t)}{\alpha} * e^{-\lambda_{m} t}
\end{aligned}\right.
$$

We substitute (8) into (7) to get,

$$
y(t)=[1,1, \ldots, 1]\left[\begin{array}{c}
x_{0}(t) \\
x_{1}(t) \\
\ldots \\
x_{M}(t)
\end{array}\right]=[1,1, \ldots, 1] x(t)
$$

To this point, we have established the dynamic battery discharge model as

$$
\begin{aligned}
& \dot{x}(t)=A x(t)+b i(t) \\
& y(t)=c x(t)
\end{aligned}
$$

where

$$
A=\operatorname{diag}\left[0,-\lambda_{1}, \cdots,-\lambda_{M}\right]_{(M+1) \times(M+1)}
$$

$$
b=\left[\begin{array}{c}
\frac{1}{\alpha} \\
\frac{2}{\alpha} \\
\cdots \\
\frac{2}{\alpha}
\end{array}\right]_{(M+1) \times 1} c=[1,1, \cdots, 1]_{1 \times(M+1)} .
$$

In addition, we have shown that this model is input-output equivalent to the RVW model when $M \rightarrow \infty$.

\section{IMPUlsive Discharge CuRRent}

The co-design for cyber-physical systems should take advantage of the recovery effect to improve the battery efficiency. To achieve this goal, we are interested in studying different discharge profiles. In this paper, we focus on the periodic impulsive current $i(t)$, which can be expressed as

$$
\begin{cases}i(t)=u & n T \leq t \leq n T+Q \\ i(t)=0 & n T+Q \leq t \leq n T+T\end{cases}
$$

where $u$ is a constant. It can be seen that the shape of $i(t)$ depends on three parameters: the pulse current $u$, the duty ratio $R=\frac{T}{Q}$ and the length of the discharge-recovery period $T$.

The problem is now formulated as studying a linear system under impulsive control. We will connect the known results for system behaviors with battery discharge behaviors.

\section{A. Role of Internal States}

Before discussing the optimal $R$ and $T$ that minimize $y(t)$, we will study the role of internal states under the impulsive discharge. Equation (10) shows that $y(t)$ is the sum of all internal states $x_{m}(t)$. We divide the $y(t)$ into two parts,

$$
y(t)=x_{0}(t)+x^{\prime}(t) .
$$

where

$$
x^{\prime}(t)=\sum_{m=1}^{M} x_{m}(t) .
$$

The state $x_{0}(t)$ is just the integration of the discharge current, hence reflects the "effective" charge drawn from the battery. We let $t=N T+\hat{t}$ where $N$ represents the number of discharge-recovery periods for the discharge current and $\hat{t} \in[0, T]$. Using linear systems theory, it is straightforward to show that

1) During the discharging time i.e. $\hat{t} \in[0, Q]$,

$$
x_{0}(t)=\frac{u}{\alpha}(N Q+\hat{t}) .
$$

2) During the resting time i.e. $\hat{t} \in[Q, T]$,

$$
x_{0}(t)=\frac{u}{\alpha}(N Q+Q)
$$

We can study $x^{\prime}(t)$ by investigating the property of each internal state $x_{m}(t)$. Again, a direct application of linear systems theory shows that at the time $t=N T+\hat{t},(\hat{t} \in[0, T])$,

$$
x_{m}(t)=\int_{0}^{N T+\hat{t}} e^{-\lambda_{m}(N T+\hat{t}-\tau)} i(\tau) \frac{2}{\alpha} d \tau
$$

and the results are 
1) During the discharging time i.e. $\hat{t} \in[0, Q]$

$$
\begin{aligned}
& x_{m}(N T+\hat{t})=\frac{2 u}{\alpha \lambda_{m}} \\
& -e^{-\lambda_{m} \hat{t}} \frac{2 u}{\alpha \lambda_{m}} \frac{\left(e^{\lambda_{m} T}-e^{\lambda_{m} Q}\right)+e^{-\lambda_{m} N T}\left(e^{\lambda_{m} Q}-1\right)}{e^{\lambda_{m} T}-1} .
\end{aligned}
$$

2) During the resting period i.e. $\hat{t} \in[Q, T]$

$$
\begin{aligned}
& x_{m}(N T+\hat{t}) \\
& =e^{-\lambda_{m} \hat{t}} \frac{2 u}{\alpha \lambda_{m}}\left(e^{\lambda_{m} Q}-1\right)\left(\frac{1-e^{-\lambda_{m} N T}}{e^{\lambda_{m} T}-1}+1\right) .
\end{aligned}
$$

It is also known that within the period of $[N T, N T+T]$, $x_{m}(t)$ increases monotonically during the discharging time and decreases monotonically during the resting time. Hence $x_{m}(t)$ oscillates in each period. The average of $x_{m}(t)$ within $[N T, N T+T]$ can be computed by

$$
\begin{aligned}
O_{m}(t) & =\frac{\int_{N T}^{N T+T} x_{m}(t) d t}{T} \\
& =\frac{2 u R}{\alpha \lambda_{m}}-\frac{2 u}{\alpha \lambda_{m}^{2} T}\left(e^{\lambda_{m} Q}-1\right) e^{-\lambda_{m}(N+1) T} .
\end{aligned}
$$

\section{B. The Switching Steady State}

The model approaches a switching steady state when $N T \rightarrow \infty$ and $e^{-\lambda_{m} N T} \rightarrow 0$. At this steady state, since $Q=R T$, we have,

$$
\begin{aligned}
& x_{m}(N T+\hat{t})_{\mathrm{st}} \\
& =\frac{2 u}{\alpha \lambda_{m}}-e^{-\lambda_{m} \hat{t}} \frac{2 u}{\alpha \lambda_{m}} \frac{\left(e^{\lambda_{m} T}-e^{\lambda_{m} R T}\right)}{e^{\lambda_{m} T}-1} \quad \hat{t} \in[0, Q], \\
& x_{m}(N T+\hat{t})_{\mathrm{st}} \\
& =e^{-\lambda_{m} \hat{t}} \frac{2 u}{\alpha \lambda_{m}}\left(e^{\lambda_{m} R T}-1\right)\left(\frac{1}{e^{\lambda_{m} T}-1}+1\right) \quad \hat{t} \in[Q, T], \\
& O_{m}(t)_{\mathrm{st}}=\frac{2 u R}{\alpha \lambda_{m}} .
\end{aligned}
$$

We use $S_{m}$ to denote the time for $O_{m}(t)$ to reach a fixed neighborhood around the steady state value. $S_{m}$ depends only on $\lambda_{m}$. Since $\lambda_{m}$ is a physical parameter of the battery, the transient time $S_{m}$ is fixed for a given battery. Since $x^{\prime}(t)$ is the sum of $x_{m}(t)$, the transient time for $x^{\prime}(t)$ is defined by $S=\max \left\{S_{1}, S_{2}, \cdots, S_{M}\right\}$, which is also determined by the battery.

\section{Optimal And Adaptive Discharge Profiles}

The $x^{\prime}(t)$ in the state space model represents the wasted electrolytes that can not be drawn by the outside current. Thus to maximize battery efficiency, we need to minimize $x^{\prime}(t)$. From the above analysis, we know that $x^{\prime}(t)$ will go through a fixed transient time $S$ to approach the steady state. However, the larger the averaged current is, the shorter the battery lifetime $L$ is. In certain situations, the battery may fail even before $x^{\prime}(t)$ reaches the steady state, i.e. $S>L$. For example, the two plots in Fig. 1 are simulated under the same battery model $\alpha=40375 \lambda_{m}=(0.273)^{2} \mathrm{~m}^{2}$. and the same discharging profile $R=0.5, T=4 \mathrm{~min}$. The impulse current in the left plot has $u_{1}=222.7 \mathrm{~mA}$ while the impulse current in the right plot has $u_{2}=1000 \mathrm{~mA}$. The battery lifetime $L$ is the time when $y(t)$ reaches 1 . In the left plot, we see that $L>S$ and the $x^{\prime}(t)$ can reach the steady state before time $L$. In the right plot, $L<S$ and the battery fails before $x^{\prime}(t)$ getting close to the steady state.
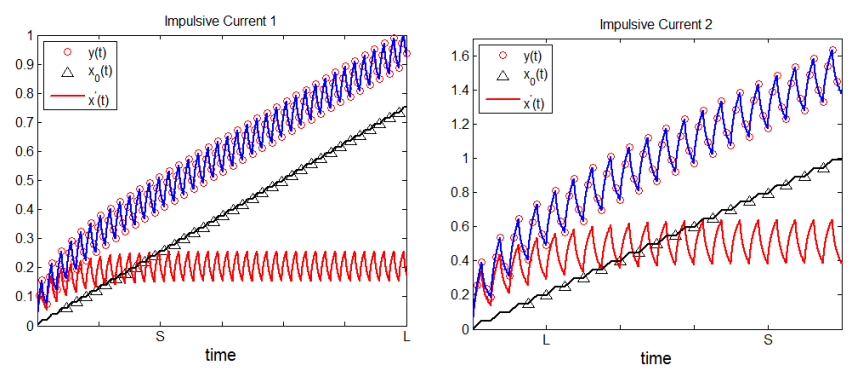

Fig. 1. Two possibilities for battery discharge. The total discharge output $y(t)$, the effective discharge $x_{0}(t)$, and the wasted discharge $x^{\prime}(t)$ are plotted in both plots. $L$ is the time when $y(t)=1$ and $S$ is the time when $x^{\prime}(t)$ is near the steady state. The discharge current for the left plot is smaller than the right plot. Left, $S<L$; right, $S>L$.

We assume that the level $u$ of the discharge current is fixed. We focus on the case where $x^{\prime}(t)$ approaches the steady state before the failure of battery, i.e. $S<L$. At the steady state, $x^{\prime}(N T+\hat{t})_{\mathrm{st}}$ is periodic.

\section{A. Optimal Discharge Profiles}

In this section, we determine the optimal $R$ and $T$ that minimize the steady state $x^{\prime}(t)$ at times $N T$ and $N T+$ $Q$. As we discussed in the previous section, $x(N T)_{\mathrm{st}}$ is the minimum during one period and $x(N T+Q)_{\text {st }}$ is the maximum during one period. We first determine the optimal duty $R$ given that $T$ is fixed. Then we determine the optimal period $T$ given the optimal duty $R_{\mathrm{opt}}$.

Given fixed period $T,(18)$ and (19) are all monotonic increasing with respect to $R>0$. Thus, decreasing $R$ will decrease the $x_{m}(t)_{\text {st }}$ for all $t$. The same property holds for $x^{\prime}(t)_{\text {st. }}$. The optimal duty cycle is the minimum possible value for $R$ determined by other practical constraints.

Next, we determine the optimal $T$ given $R=R_{\text {opt }}$ fixed. Regarding the average state $O_{m}(t)$, we have

$$
O_{m}(t)_{\mathrm{st}}=\frac{2 u R_{\mathrm{opt}}}{\alpha \lambda_{m}} .
$$

Apparently, $O_{m}(t)_{\text {st }}$ is fixed by $R_{\text {opt }}$ and not affected by $T$. Hence $x_{m}(t)_{\text {st }}$ will oscillate around the same level as $T$ changes. The same property holds for $x^{\prime}(t)_{\mathrm{st}}$.

Our conclusion is that the larger $T$ is, the more impulsive $x^{\prime}(t)_{\text {st }}$ is. The justification to the conclusion is as follows. First, consider the minimum value for $x_{m}$ during one period, according to (18), we have

$$
x_{m}(N T)_{\mathrm{st}}=\frac{2 u}{\alpha \lambda_{m}} \frac{e^{\lambda_{m} R_{\mathrm{opt}} T}-1}{e^{\lambda_{m} T}-1},
$$

which can now be viewed as a function of $T$. Taking the derivative of $x_{m}(N T)_{\mathrm{st}}$ with respect to $T$ and after 
simplification, we have

$$
\begin{aligned}
& \frac{d x_{m}(N T)_{\mathrm{st}}}{d T}=\frac{2 u}{\alpha} \frac{e^{\lambda_{m} T}}{\left(e^{\lambda_{m} T}-1\right)^{2}} \times \\
& \left(1-R_{\mathrm{opt}} e^{\lambda_{m} R_{\mathrm{opt}} T-\lambda_{m} T}-\left(1-R_{\mathrm{opt}}\right) e^{\lambda_{m} R_{\mathrm{opt}} T}\right) .
\end{aligned}
$$

Define $H(T)$ to be

$$
H(T)=1-R_{\mathrm{opt}} e^{\lambda_{m}\left(R_{\mathrm{opt}}-1\right) T}-\left(1-R_{\mathrm{opt}}\right) e^{\lambda_{m} R_{\mathrm{opt}} T} .
$$

Then, one can verify that

$$
H(0)=0 \quad \frac{d H(T)}{d T}<0
$$

which implies that $H(T)<0$ for all $T>0$. Moreover, plug (24) back into (23),

$$
\frac{d x_{m}(N T)_{\mathrm{st}}}{d T}=\frac{2 u}{\alpha} \frac{e^{\lambda_{m} T}}{\left(e^{\lambda_{m} T}-1\right)^{2}} H(T) .
$$

We must have that

$$
\frac{d x_{m}(N T)_{\mathrm{st}}}{d T}<0 \quad \text { if } T>0
$$

which means that the function $x_{m}(N T)_{\text {st }}$ is monotone decreasing whenever $T>0$. The longer the period $T$, the smaller $x_{m}(N T)_{\text {st }}$ is. The same property holds for $x^{\prime}(N T)_{\text {st. }}$.

Now consider $x_{m}(N T+Q)_{\text {st }}$, the maximum value for $x_{m}$ during one discharge-recovery period. According to equation (19), we have

$$
x_{m}(N T+Q)_{\mathrm{st}}=\frac{2 u}{\alpha \lambda_{m}}\left(1-e^{-\lambda_{m} R_{\mathrm{opt}} T}\right) \frac{e^{\lambda_{m} T}}{e^{\lambda_{m} T}-1} .
$$

By similar arguments made for $x_{m}(N T)_{\mathrm{st}}$, we can show that

$$
\frac{d x_{m}(N T+Q)_{\mathrm{st}}}{d T}>0 \quad \text { if } T>0
$$

which means that the function $x_{m}(N T+Q)_{\text {st }}$ is monotone increasing whenever $T>0$. The longer the period $T$, the larger $x_{m}(N T+Q)_{\text {st }}$ is. The same property holds for $x^{\prime}(N T+Q)_{\text {st }}$.

Thus, the following proposition has been proved.

Proposition 1: Consider the dynamic battery model under a square wave impulsive discharge current. In the steady state, the average state $O_{m}(t)_{\mathrm{st}}$ is determined by (21), which does not depend on the discharge-recovery period $T$. For the internal state $x_{m}(t)_{\text {st }}$ where $m=1,2, \ldots, M$, the difference between its maximum and its minimum during one period $T$ increases monotonically as $T$ increases.

\section{B. An Adaptive Discharge Strategy}

In this section, we design an adaptive discharge strategy that improves the battery efficiency as follows

1) Select the minimum $R$ and keep it fixed during the lifetime of the battery.

2) Use large $T$ initially, then switch to smaller $T$ when approaching the end of battery life.

We will determine what the switching time and the adaptive period should be.
For simplicity, we only discuss a two-step adaptive strategy, in which the period is only changed once. Similar arguments can be made for multi-step strategies. Suppose the initial discharge profile is selected as $T_{1}, R$ and the lifetime of a battery under this discharge can be written as $L_{1}=N_{1} T_{1}+\hat{t}_{1}$. We assume that $S<L$. We have $Q_{1}=R T_{1}$ and

$$
y\left(L_{1}\right)=x_{0}\left(L_{1}\right)_{\mathrm{st}}+x^{\prime}\left(L_{1}\right)_{\mathrm{st}}=1 .
$$

During a discharge-recovery period, $y(t)$ first increases and then decreases. Therefore,

$$
\left\{\begin{array}{l}
y\left(\left(N_{1}-1\right) T_{1}+Q_{1}\right)<1 \\
y\left(N_{1} T_{1}+Q_{1}\right)>1
\end{array}\right.
$$

Substitute (12) and (18) into (28), we have

$\left\{\begin{array}{l}N_{1}+\sum_{m=1}^{M} \frac{2}{\lambda_{m} Q_{1}}\left(1-e^{-\lambda_{m} Q_{1}}\right) \frac{e^{\lambda_{m} T_{1}}}{e^{\lambda_{m} T_{1}}-1}<\frac{\alpha}{u Q_{1}} \\ N_{1}+1+\sum_{m=1}^{M} \frac{2}{\lambda_{m} Q_{1}}\left(1-e^{-\lambda_{m} Q_{1}}\right) \frac{e^{\lambda_{m} T_{1}}}{e^{\lambda_{m} T_{1}}-1}>\frac{\alpha}{u Q_{1}} .\end{array}\right.$

Solve the above equations,

$$
N_{1}=\left\lfloor\frac{\alpha}{u Q_{1}}-\sum_{m=1}^{M} \frac{2}{\lambda_{m} Q_{1}}\left(1-e^{-\lambda_{m} Q_{1}}\right) \frac{e^{\lambda_{m} T_{1}}}{e^{\lambda_{m} T_{1}}-1}\right\rfloor .
$$

The time to switch to a different period is then $N_{1} T_{1}$.

We now determine the new discharge-recovery period $T_{2}$. Applying (12) and (18) into (27), we have

$$
\begin{aligned}
& \hat{t}_{1}-e^{-\lambda_{m} \hat{t}_{1}} \sum_{m=1}^{M} \frac{2}{\lambda_{m}} \frac{e^{\lambda_{m} T_{1}}-e^{\lambda_{m} Q_{1}}}{e^{\lambda_{m} T_{1}}-1}+\sum_{m=1}^{M} \frac{2}{\lambda_{m}} \\
& +N_{1} Q_{1}-\frac{\alpha}{u}=0 .
\end{aligned}
$$

Define the left side of the above equation to be $f\left(\hat{t}_{1}\right)$, one can verify that

$$
f(0)<0 \quad \frac{d f\left(\hat{t}_{1}\right)}{d \hat{t}_{1}}>0 .
$$

Therefore there exists unique $\hat{t}_{1}$ satisfying the equation

$$
f\left(\hat{t}_{1}\right)=0 \text {. }
$$

The new period is then $T_{2}=\frac{\hat{t}_{1}}{R}$.

A new time $\hat{t}_{2}$ can then be calculated based on $R$ and $T_{2}$ and the battery life will be prolonged by $\hat{t}_{2}$. The battery life can be further prolonged by allowing multiple switching to shorter discharge-recovery periods.

\section{Simulation Results}

We compare simulation results for a battery with the following parameters:

$$
I=50 \mathrm{~mA} \quad \alpha=40375 \quad \lambda_{m}=(0.273)^{2} \mathrm{~m}^{2}
$$

under different discharge profiles.

The influence of the duty ratio $R$ and the time period $T$ is shown in Fig. 2. In the left plot, the time period is fixed to be $T=20$ minutes. We can see that the smaller the duty ratio $R$, the smaller $x^{\prime}(t)$ is, i.e. the less unavailable species and thus the more efficient the battery is. In the right plot, the 
duty ratio is fixed to be $R=0.5$. It indicates that the larger the time period $T$, the more impulsive $x^{\prime}(t)$ is, i.e. the less unavailable species in the battery at the end of each period but more overshoot during the period. These are consistent with our analysis.
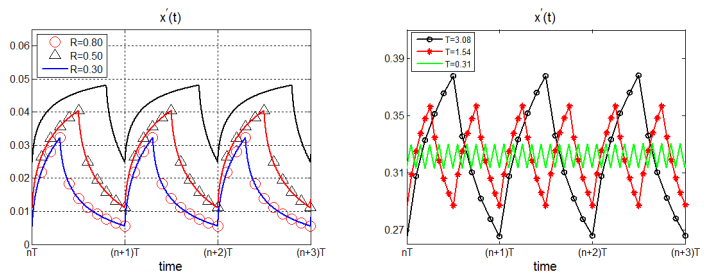

Fig. 2. Battery capacity under different duty ratios and time periods

In Fig.3, we show the battery efficiency improved by adaptive strategy. The physical parameter of the battery is

$$
I=300 \mathrm{~mA} \quad \alpha=40375 \quad \lambda_{m}=(0.273)^{2} \mathrm{~m}^{2} .
$$

Initially, we choose $T_{1}=8$ minutes, $R=0.5$. We compute the value $N_{1}=26$ and $\hat{t}_{1}=3.3$. Then, at the time $N_{1} T_{1}=$ 208 , we switch to another discharge profile $T_{2}=\frac{\hat{t}_{1}}{R_{1}}$. We can see that the online running time of the battery is prolonged by the adaptive strategy.

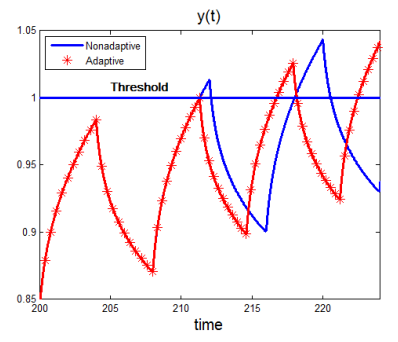

Fig. 3. Adaptive Strategy prolongs battery life

\section{CONCLUSIONS}

We introduce a dynamic battery discharge model that is input-output equivalent to the RVW model. Since the accuracy of RVW model has been verified by experimental data, it is reasonable to conclude that the dynamic battery discharge model inherits such accuracy. The states of this model represent the instantaneous discharge/recovery effects within the battery. Using this dynamic model, the battery can be viewed as a linear system under the control $i(t)$. Optimal and adaptive $i(t)$ can be determined analytically without seeking numerical methods. The battery model and discharge strategy will be applied to mobile sensor networks [21]-[23] where battery aware control laws and scheduling strategies increases operation time.

\section{REFERENCES}

[1] C.-F. Chiasserini and R. Rao, "Energy efficient battery management," IEEE Journal on Selected Areas in Communications, vol. 19, no. 7, pp. 1235-1245, 2001.

[2] R. Rao, S. Vrudhula, and D. N. Rakhmatov, "Battery modeling for energy-aware system design," Computer, vol. 36, no. 12, pp. 77-87, 2003.
[3] D. Rakhmatov, S. Vrudhula, and D. A. Wallach, "A model for battery lifetime analysis for organizing applications on a pocket computer," IEEE Transactions on VLSI Systems, vol. 11, no. 6, pp. 1019-1030, 2003.

[4] F. Zhang, K. Szwaykowska, V. Mooney, and W. Wolf, "Task scheduling for control oriented requirements for cyber-physical systems," in Proc. of 29th IEEE Real-Time Systems Symposium (RTSS 2008), Barcelona, Spain, 2008, pp. 47-56.

[5] F. Xia and Y. Sun, "Control-scheduling codesign: A perspective on integrating control and computing," in Dynamics of Continuous, Discrete and Impulsive Systems - Series B: Applications and Algorithms, Special Issue on ICSCA'06. Rome, Italy: Watam Press, 2006, pp. 1352-1358.

[6] L. Sha, T. Abdelzaher, K.-E. Årzén, A. Cervin, T. Baker, A. Burns, G. Buttazzo, M. Caccamo, J. Lehoczky, and A. K. Mok, "Real time scheduling theory: A historical perspective," Real-Time Systems, vol. 28, no. 2-3, pp. 101-155, 1994.

[7] X. Liu, Q. Wang, W. He, M. Caccamo, and L. Sha, "Optimal realtime sampling rate assignment for wireless sensor networks," $A C M$ Transactions on Sensor Networks (TOSN), vol. 2, no. 2, pp. 263-295, 2006

[8] T. Chantem, X. S. Hu, and M. Lemmon, "Generalized elastic scheduling," in Proc. 27th RTSS, 2006.

[9] F. Yao, A. Demers, and S. Shenker, "A scheduling model for reduced CPU energy," in Proc. IEEE 36th Annual FOCS, 1995, pp. 374-382.

[10] T. Ishihara and H. Yasuura, "Voltage scheduling problems for dynamically variable voltage processors," in Proc. IEEE ISLPED 1998, 1998, pp. 197-202.

[11] A. Azevedo, I. Issenin, R. Cornea, R. Gupta, N. Dutt, A. Viedenbaum, and A. Nicolau, "Profile-based dynamic scheduling using program checkpoints," in Proc. IEEE DATE 2002, 2002, pp. 168-178.

[12] R. Jejurikar, C. Pereira, and R. Gupta, "Leakage aware dynamic voltage scaling for real-time embedded systems," in Proc. IEEE DATE 2004, 2004, pp. 275-281.

[13] Z. Ren, B. H. Krogh, and R. Marculescu, "Hierarchical adaptive dynamic power management," IEEE Transactions on Computers, vol. 54, no. 4, pp. 409-420, 2005.

[14] M. Doyle, T. Fuller, and J. Newman, "Modeling of galvanostatic charge and discharge of the lithium/polymer/insertion cell," J. Electrochemical Soc., vol. 140, no. 6, pp. 1526-1533, 1995.

[15] D. Linden and T. Reddy, Handbook of Batteries, 3rd ed. McGrawHill, 2001.

[16] M. Chen and G. A. Rincon-Mora, "Accurate electrical battery model capable of predicting runtime and $i v$ performance," IEEE transactions on energy conversion, vol. 21, pp. 504-511, 2006.

[17] M. Pedram and Q. Wu, "Battery-powered digital CMOS design," IEEE Transactions on VLSI Systems, vol. 10, no. 5, pp. 601-607, 2002.

[18] M. S. Branicky, "Multiple Lyapunov functions and other analysis tools for switched and hybrid systems," IEEE Trans. Automat. Cont., vol. 43, pp. 475-482, 1998.

[19] D. Liberzon and A. S. Morse, "Benchmark problems in stability and design of switched systems," IEEE Control Systems Magazine, pp. 59 $-70,1999$.

[20] J. P. Hespanha and A. S. Morse, "Stability of switched systems with average dwell-time," in Proc. of 38th IEEE Conference on Decision and Control, Phoenix, AZ, USA, 1999, pp. $2655-2660$.

[21] J. Cortés, S. Martínez, T. Karatas, and F. Bullo, "Coverage control for mobile sensing networks," IEEE Transactions on Robotics and Automation, vol. 20, no. 2, pp. 243-255, 2004.

[22] F. Zhang and N. E. Leonard, "Coordinated patterns of unit speed particles on a closed curve," Systems and Control Letters, vol. 56, no. 6, pp. 397-407, 2007.

[23] F. Zhang, D. M. Fratantoni, D. Paley, J. Lund, and N. E. Leonard, "Control of coordinated patterns for ocean sampling," International Journal of Control, vol. 80, no. 7, pp. 1186-1199, 2007. 